Honam Mathematical J. 33 (2011), No. 3, pp. 419-424

http://dx.doi.org/10.5831/HMJ.2011.33.3.419

\title{
COMPACTNESS OF A SUBSPACE OF THE ZARISKI TOPOLOGY ON $\operatorname{SPEC}(D)$
}

\author{
Gyu Whan Chang
}

\begin{abstract}
Let $D$ be an integral domain, $\operatorname{Spec}(D)$ the set of prime ideals of $D$, and $X$ a subspace of the Zariski topology on $\operatorname{Spec}(D)$. We show that $X$ is compact if and only if given any ideal $I$ of $D$ with $I \nsubseteq P$ for all $P \in X$, there exists a finitely generated ideal $J \subseteq I$ such that $J \nsubseteq P$ for all $P \in X$. We also prove that if $D=\cap_{P \in X} D_{P}$ and if $*$ is the star-operation on $D$ induced by $X$, then $X$ is compact if and only if $*_{f}-\operatorname{Max}(D) \subseteq X$. As a corollary, we have that $t-\operatorname{Max}(D)$ is compact and that $\mathcal{P}(D)=\{P \in \operatorname{Spec}(D) \mid P$ is minimal over $(a: b)$ for some $a, b \in D\}$ is compact if and only if $t-\operatorname{Max}(D) \subseteq \mathcal{P}(D)$.
\end{abstract}

\section{Introduction}

Let $D$ be an integral domain, and let $\operatorname{Spec}(D)$ be the set of prime ideals of $D$. For each subset $E$ of $D$, let $V(E)$ denote the set of all prime ideals of $D$ which contain $E$. Then the sets $V(E)$ satisfy the axioms for the closed sets in a topological space. The resulting topology is called the Zariski topology on $\operatorname{Spec}(D)$. For each $f \in D$, let $X_{f}=$ $\operatorname{Spec}(D) \backslash V(\{f\})$; then $X_{f}=\{P \in \operatorname{Spec}(D) \mid f \notin P\}$ and $X_{f}$ is an open subset of $\operatorname{Spec}(D)$. Obviously, $\left\{X_{f} \mid f \in D\right\}$ forms a basis of open sets for $\operatorname{Spec}(D)$. Note that if $X$ is a nonempty set of prime ideals of $D$, then the collection $\left\{X_{f} \cap X \mid f \in D\right\}$ forms a basis of open sets for the subspace topology $X$ of $\operatorname{Spec}(D)$. Recall that a topological space $T$ is said to be compact if every open covering of $T$ contains a finite subcollection that also covers $T$.

If $\Lambda$ is a nonempty set of elements of $D$ such that $\operatorname{Spec}(D)=\cup_{f \in \Lambda} X_{f}$, then $(\{f \mid f \in \Lambda\})=D$, and hence $1=\sum_{i=1}^{n} f_{i} g_{i}$ for some $f_{i} \in \Lambda$ and

Received August 2, 2011. Accepted August 10, 2011.

2000 Mathematics Subject Classification. 13A15, 13G05, 16 W99.

Key words and phrases. Zariski topology, subspace topology, compactness, $*_{f}$ $\operatorname{Max}(D), \mathcal{P}(D)$. 
$g_{i} \in D$. Thus $\operatorname{Spec}(D)=X_{f_{1}} \cup \cdots \cup X_{f_{n}}$, and this implies that $\operatorname{Spec}(D)$ is a compact topological space (cf. [2, page 12]). Let $X$ be a subspace topology of the Zariski topology on $\operatorname{Spec}(D)$. In this paper, we show that $X$ is compact if and only if, given any ideal $I$ of $D$ with $I \nsubseteq P$ for all $P \in X$, there exists a finitely generated ideal $J \subseteq I$ such that $J \nsubseteq P$ for all $P \in X$. As a corollary, we have that if $*$ is a star-operation on $D$, then $*_{f}$-Max $(D)$ is compact. (Definitions related to star-operations will be reviewed at the end of this section.) We also prove that if $D=\cap_{P \in X} D_{P}$ and if $*$ is the star-operation on $D$ induced by $X$, then $X$ is compact if and only if $*_{f}-\operatorname{Max}(D) \subseteq X$. Let $\mathcal{P}(D)=\{P \in \operatorname{Spec}(D) \mid P$ is minimal over $(a: b)$ for some $a, b \in D\}$ and $x$ be an indeterminate over $D$. Also, we prove that $\mathcal{P}(D)$ is compact if and only if $t$-Max $(D) \subseteq \mathcal{P}(D)$ and that $X$ is compact if and only if $\{P[x] \mid P \in X\}$ is compact.

Let $K$ be the quotient field of an integral domain $D$, and let $\mathbf{F}(D)$ be the set of nonzero fractional ideals of $D$. A star-operation $*$ on $D$ is a mapping $I \mapsto I^{*}$ from $\mathbf{F}(D)$ into $\mathbf{F}(D)$ such that (i) $(a D)^{*}=a D$ and $(a I)^{*}=a I^{*}$, (ii) $I \subseteq I^{*}$, and if $I \subseteq J$, then $I^{*} \subseteq J^{*}$, and (iii) $\left(I^{*}\right)^{*}=I^{*}$ for all $0 \neq a \in K$ and all $I, J \in \mathbf{F}(D)$. An $I \in \mathbf{F}(D)$ is called a $*$-ideal if $I^{*}=I$. For $I \in \mathbf{F}(D)$, let $I^{*_{f}}=\cup J^{*}$, where $J$ ranges over all nonzero finitely generated subideals of $I$. It is well known that $*_{f}$ is also a star-operation on $D$. A star-operation is said to be of finite type if $*=*_{f}$. Let $*-\operatorname{Max}(D)$ denote the set of $*$-ideals of $D$ maximal among proper integral $*$-ideals of $D$. It is well known that $*_{f}-\operatorname{Max}(D) \neq \emptyset$ if $D$ is not a field; each proper integral $*_{f}$-ideal is contained in a maximal $*_{f}$-ideal; a maximal $*_{f}$-ideal is a prime ideal; each prime ideal minimal over a $*_{f}$-ideal is a $*_{f}$-ideal; and $D=\cap_{P \in *_{f}-\operatorname{Max}(D)} D_{P}$. Let $X$ be a nonempty set of prime ideals of $D$ such that $D=\bigcap_{P \in X} D_{P}$. For each $I \in \mathbf{F}(D)$, define $I^{*}=\cap_{P \in X} I D_{P}$; then $*$ is a star-operation on $D[1$, Theorem 1]. We call $*$ the star-operation induced by $X$.

The most well-known star-operations are the $v-, t$-, and $d$-operations. The $v$-operation is defined by $I_{v}=\left(I^{-1}\right)^{-1}$, where $I^{-1}=\{a \in K \mid a I \subseteq$ $D\}$, the $t$-operation is defined by $t=v_{f}$, and the $d$-operation is the identity function on $\mathbf{F}(D)$, i.e., $I_{d}=I$ for all $I \in \mathbf{F}(D)$. It is well known that $I=I_{d} \subseteq I^{*_{f}} \subseteq I_{t} \subseteq I_{v}$ for all $I \in \mathbf{F}(D)$. For more on star-operations, see $[3, \S 32$ and $\S 34]$.

\section{Main Results}

Throughout $D$ is an integral domain. Let $\mathcal{P}(D)=\{P \in \operatorname{Spec}(D) \mid P$ is minimal over $(a: b)$ for some $a, b \in D\}$. In [6, Lemma 3.1], Papick 
showed that $\mathcal{P}(D)$ is compact if and only if, given any ideal $I$ of $D$ with $I \nsubseteq P$ for all $P \in \mathcal{P}(D)$, there exists a finitely generated ideal $J \subseteq I$ such that $J \nsubseteq P$ for all $P \in \mathcal{P}(D)$.

We first generalize Papick's result to an arbitrary nonempty set of prime ideals of $D$, which is the main result of this paper.

Theorem 1. Let $\emptyset \neq X \subseteq \operatorname{Spec}(D)$. Then $X$ is compact if and only if, given any ideal $I$ of $D$ with $I \nsubseteq P$ for all $P \in X$, there exists a finitely generated ideal $J \subseteq I$ such that $J \nsubseteq P$ for all $P \in X$.

Proof. $(\Rightarrow)$ For each $P \in X$, choose $a_{p} \in I \backslash P$, and note that

$$
X=\cup_{P \in X}\left(X_{a_{p}} \cap X\right),
$$

where $X_{a_{p}}=\left\{P^{\prime} \in \operatorname{Spec}(D) \mid a_{p} \notin P^{\prime}\right\}$. Note also that each $X_{a_{p}} \cap X$ is an open set in $X$; so by assumption, there are $a_{p_{1}}, \ldots, a_{p_{k}}$ such that

$$
X=\left(X_{a_{p_{1}}} \cap X\right) \cup \cdots \cup\left(X_{a_{p_{k}}} \cap X\right) .
$$

Let $J=\left(a_{p_{1}}, \ldots, a_{p_{k}}\right)$; then obviously, $J \subseteq I$ and $J \nsubseteq P$ for all $P \in X$.

$(\Leftarrow)$ It suffices to show that any open cover of $X$ consisting of basic open sets has a finite subcover. Suppose that $X=\cup_{\alpha \in \Lambda}\left(X_{a_{\alpha}} \cap X\right)$, where $\left\{X_{a_{\alpha}} \mid \alpha \in \Lambda\right\}$ is a family of basic open sets for $\operatorname{Spec}(D)$, and let $I=\left(\left\{a_{\alpha} \mid \alpha \in \Lambda\right\}\right)$. Then $I \nsubseteq P$ for all $P \in X$, and hence there are some $\alpha_{1}, \ldots, \alpha_{s} \in \Lambda$ such that $J=\left(a_{\alpha_{1}}, \ldots, a_{\alpha_{s}}\right) \nsubseteq \subseteq P$ for all $P \in X$ by assumption. Thus $X=\left(X_{a_{\alpha_{1}}} \cap X\right) \cup \cdots \cup\left(X_{a_{\alpha_{s}}} \cap X\right)$.

Corollary 2. Let $X$ be a nonempty set of prime ideals of $D$ such that (i) there are no containment relations among distinct members of $X$ and (ii) each prime ideal of $D$ contained in $\cup_{P \in X} P$ is contained in some $P \in X$. Then $X$ is compact. In particular, the set of maximal ideals of $D$ is compact.

Proof. Let $I$ be an ideal of $D$ such that $I \nsubseteq P$ for all $P \in X$. Let $S=D \backslash \cup_{P \in X} P$, and note that $\left\{P D_{S} \mid P \in X\right\}$ is the set of maximal ideals of $D_{S}\left[3\right.$, Proposition 4.8]. Since $I \nsubseteq P$, we have $I D_{S} \nsubseteq P D_{S}$ for all $P \in X$; so $I D_{S}=D_{S}$. Hence $1=\sum_{i=1}^{n} f_{i} \frac{d_{i}}{s_{i}}$ for some $f_{i} \in I, d_{i} \in D$, and $s_{i} \in S$. So if we set $s=s_{1} \cdots s_{n}$, then $s \in I \backslash \cup_{P \in X} P$, and hence $s D \subseteq I$ with $s D \nsubseteq P$ for all $P \in X$. Thus $X$ is compact by Theorem 1.

Corollary 3. If $*$ is a star-operation on $D$, then $*_{f}-\operatorname{Max}(D)$ is compact. In particular, $t-\operatorname{Max}(D)$ is compact. 
Proof. Let $I$ be an ideal of $D$ with $I \nsubseteq P$ for all $P \in *_{f}-\operatorname{Max}(D)$. Then $I^{*}=D$, and since $*_{f}$ is of finite type, there exists a finitely generated ideal $J \subseteq I$ with $J^{*_{f}}=D$. Again, since $*_{f}$ is of finite type, $J \nsubseteq P$ for each $P \in *_{f}-\operatorname{Max}(D)$. Thus $*_{f}-\operatorname{Max}(D)$ is compact by Theorem 1.

Tang proved that, for a finitely generated ideal $I$ of $D, I \subseteq P$ for some $P \in \mathcal{P}(D)$ if and only if $I_{v} \subsetneq D[7$, Theorem E]. This was improved by Zafrullah [8, Theorem 1] as follows: Let $\left\{M_{i}\right\}_{i \in \Lambda}$ be a set of prime ideals of $D$ such that $D=\cap D_{M_{i}}$. If $I$ is an ideal of $D$ with $I_{v} \subsetneq D$, then $I_{v}$, and hence $I$, is contained in at least one $M_{i}$.

An integral domain $D$ is a $P$-domain if $D_{P}$ is a valuation domain for each $P \in \mathcal{P}(D)$, while $D$ is a Prüfer $v$-multiplication domain $(\mathrm{P} v \mathrm{MD})$ if each nonzero finitely generated ideal $I$ of $D$ is $t$-invertible, i.e., $\left(I I^{-1}\right)_{t}=$ $D$. It is well known that $D$ is a $\mathrm{P} v \mathrm{MD}$ if and only if $D_{P}$ is a valuation domain for each $P \in t-\operatorname{Max}(D)$; so a $\mathrm{P} v \mathrm{MD}$ is a $\mathrm{P}$-domain. Let $D$ be a $\mathrm{P}$-domain that is not a $\mathrm{P} v \mathrm{MD}$ [5, Example 2.1]. Then there is a maximal $t$-ideal $Q$ of $D$ such that $Q \notin \mathcal{P}(D)$; in particular, $Q \nsubseteq P$ for each $P \in \mathcal{P}(D)$. Thus $I$ being finitely generated is necessary for [7, Theorem E] and Zafrullah's result does not hold for an ideal $I$ with $I_{t} \subsetneq D$. The next result shows that if $\left\{M_{i}\right\}$ is compact, then each ideal $I$ of $D$ with $I_{t} \subsetneq D$ is contained in at least one $M_{i}$.

Corollary 4. Let $X \subseteq \operatorname{Spec}(D)$ such that $D=\cap_{P \in X} D_{P}$, and let $*$ be the star-operation on $D$ induced by $X$. Then $X$ is compact if and only if $*_{f}-\operatorname{Max}(D) \subseteq X$. In this case, each $P \in t-\operatorname{Max}(D)$ is contained in at least one $Q \in X$.

Proof. Suppose that $X$ is compact, and let $Q$ be a maximal $*_{f}$-ideal of $D$. Assume to the contrary that $Q \nsubseteq \subseteq P$ for all $P \in X$. By Theorem 1 , there exists a finitely generated ideal $J \subseteq Q$ such that $J \nsubseteq P$ for all $P \in X$. Then $J^{*}=\cap_{P \in X} J D_{P}=\cap_{P \in X} D_{P}=D$, and hence $D=J^{*} \subseteq$ $Q^{* f}=Q \subsetneq D$, a contradiction. So $Q \subseteq P$ for some $P \in X$. Note that if $P^{\prime} \in X$, then $P^{\prime} \subseteq\left(P^{\prime}\right)^{*_{f}} \subseteq\left(P^{\prime}\right)^{*}=P^{\prime}$; so $\left(P^{\prime}\right)^{*_{f}}=\left(P^{\prime}\right)^{*}=P^{\prime}$. Thus $Q=P$. Conversely, assume that $*_{f}-\operatorname{Max}(D) \subseteq X$, and let $I$ be an ideal of $D$ such that $I \nsubseteq P$ for all $P \in X$. Then by assumption, $I^{*} f=D$, and since $*_{f}$ is of finite type, there exists a finitely generated ideal $J \subseteq I$ such that $J^{* f}=D$. As we note, each $P \in X$ is a $* f_{\text {-ideal, and hence }}$ $J \nsubseteq P$ for all $P \in X$. Thus $X$ is compact by Theorem 1 .

For the "in this case" part, note that $I \subseteq I^{*_{f}} \subseteq I_{t}$ for all $I \in$ $\mathbf{F}(D)$. Hence each $Q^{\prime} \in t$-Max $(D)$ is a $*_{f}$-ideal, and thus $Q^{\prime} \subseteq P^{\prime} \in *_{f^{-}}$ $\operatorname{Max}(D) \subseteq X$. 
The result of Corollary 4 shows that if there are no containment relations among distinct members of $X$, then $X$ is compact if and only if $X=*_{f}-\operatorname{Max}(D)$.

Corollary 5. 1. If $X$ is a set of prime t-ideals of $D$ with $D=$ $\cap_{P \in X} D_{P}$, then $X$ is compact if and only if $t-\operatorname{Max}(D) \subseteq X$.

2. $\mathcal{P}(D)$ is compact if and only if $t-\operatorname{Max}(D) \subseteq \mathcal{P}(D)$.

Proof. (1) If $X$ is compact, then $*_{f}-\operatorname{Max}(D) \subseteq X$ by Corollary 4 , and since each ideal in $X$ is a $t$-ideal, each maximal $*_{f}$-ideal of $D$ is a $t$-ideal, and hence $*_{f}-\operatorname{Max}(D)=t-\operatorname{Max}(D)$. Thus $t-\operatorname{Max}(D) \subseteq X$. Conversely, assume $t-\operatorname{Max}(D) \subseteq X$, and let $I$ be an ideal of $D$ such that $I \nsubseteq P$ for all $P \in X$. Then by assumption, $I_{t}=D$, and thus there is a finitely generated ideal $J \subseteq I$ with $J_{v}=D$. Since each ideal in $X$ is a $t$-ideal, $J \nsubseteq P$ for all $P \in X$. Thus $X$ is compact by Theorem 1 .

(2) Note that each prime ideal in $\mathcal{P}(D)$ is a $t$-ideal and $D=\cap_{P \in \mathcal{P}(D)} D_{P}$. Thus the result follows directly from (1).

Let $D$ be a $\mathrm{P}$-domain. If $t-\operatorname{Max}(D) \subseteq \mathcal{P}(D)$, then $D_{P}$ is a valuation domain for each $P \in t-\operatorname{Max}(D)$, and hence $D$ is a $\mathrm{P} v \mathrm{MD}$. Thus if $D$ is not a $\mathrm{P} v \mathrm{MD}$ (see [5, Example 2.1]), then $\mathcal{P}(D)$ is not compact by Corollary $5(2)$.

Corollary 6. ([6, Proposition 3.2]) Let $x$ be an indeterminate over $D$. Then $\mathcal{P}(D)$ is compact if and only if $\mathcal{P}(D[x])$ is compact.

Proof. Note that if $Q$ is a maximal $t$-ideal of $D[x]$, then either $Q \cap D=$ (0) or $Q=P[x]$ for some $P \in t-\operatorname{Max}(D)$ [4, Proposition 1.1]. Also, note that $(a: b) D[x]=(a D[x]: b D[x])$; so $t-\operatorname{Max}(D) \subseteq \mathcal{P}(D)$ if and only if $t$-Max $(D[x]) \subseteq \mathcal{P}(D[x])$. Thus the result follows from Corollary 5 .

Corollary 7. Let $\emptyset \neq X \subseteq \operatorname{Spec}(D)$ and $x$ an indeterminate over $D$.

1. $X$ is compact if and only if $\{P[x] \mid P \in X\}$ is compact.

2. If $*$ is a star-operation on $D$, then $\left\{P[x] \mid P \in *_{f}-\operatorname{Max}(D)\right\}$ is compact.

Proof. (1) Let $T=\{P[x] \mid P \in X\}$. Suppose that $X$ is compact, and let $T=\cup_{f \in \Lambda}\left(X_{f} \cap T\right)$, where $\Lambda \subseteq D[x]$ and $X_{f}=\{Q \in \operatorname{Spec}(D[x]) \mid f \notin$ $Q\}$. Put $\Gamma=\{a \in D \mid a$ is a coefficient of a polynomial $f \in \Lambda\}$. Obviously, $X=\cup_{a \in \Gamma}\left(X_{a} \cap X\right)$, where $X_{a}=\{P \in \operatorname{Spec}(D) \mid a \notin P\}$, and since $X$ is compact, there exist some $a_{1}, \ldots, a_{k} \in \Gamma$ such that $X=\left(X_{a_{1}} \cap X\right) \cup$ $\cdots \cup\left(X_{a_{k}} \cap X\right)$. Hence if $f_{i} \in \Lambda$ is such that $a_{i}$ is a coefficient of $f_{i}$, then $T=\left(X_{f_{1}} \cap T\right) \cup \cdots \cup\left(X_{f_{k}} \cap T\right)$. 
Conversely, suppose that $T$ is compact, and let $X=\cup_{a \in I}\left(X_{a} \cap X\right)$, where $I \subseteq D$ and $X_{a}=\{P \in \operatorname{Spec}(D) \mid a \notin P\}$. Clearly, $T=\cup_{a \in I}\left(X_{a} \cap\right.$ $T)$, where $X_{a}=\{Q \in \operatorname{Spec}(D[x]) \mid a \notin Q\}$, and since $T$ is compact, we have $T=\left(X_{a_{1}} \cap T\right) \cup \cdots \cup\left(X_{f_{n}} \cap T\right)$ for some $a_{1}, \ldots, a_{n} \in I$. Thus $X=\left(X_{a_{1}} \cap X\right) \cup \cdots \cup\left(X_{a_{n}} \cap X\right)$.

(2) This follows from (1) and Corollary 3.

Acknowledgement. The author would like to thank the referees for several helpful comments. This research was supported by Basic Science Research Program through the National Research Foundation of Korea(NRF) funded by the Ministry of Education, Science and Technology (2010-0007069).

\section{References}

[1] D.D. Anderson, Star-operations induced by overrings, Comm. Algebra 16(1988), $2535-2553$.

[2] M.F. Atiyah and I.G. Macdonald, Introduction to Commutative Algebra, Addison-Wesley, 1968.

[3] R. Gilmer, Multiplicative Ideal Theory, Marcel Dekker, New York, 1972.

[4] E. Houston and M. Zafrullah, On t-invertibility II, Comm. Algebra 17 (1989) $1955-1969$

[5] J. Mott and M. Zafrullah, On Prüfer v-multiplication domain, Manuscripta Math. 35(1981), 1-26.

[6] I.J. Papick, Super-primitive elements, Pacific J. Math. 105(1983), 217-226.

[7] H. Tang, Gauss Lemma, Proc. Amer. Math. Soc. 35(1972), 372-376.

[8] M. Zafrullah, The v-operation and intersections of quotient rings of integral domains, Comm. Algebra 13(1985), 1699-1712.

Gyu Whan Chang

Department of Mathematics, University of Incheon,

Incheon 406-772, Korea.

E-mail: whan@incheon.ac.kr 